\title{
RELACIÓN ENTRE TMAX Y REFLECTANCIA DE LA VITRINITA EN LAS CUENCAS DE ANTE-ARCO DEL SUR-OESTE DEL ECUADOR
}

\section{Relationship between Tmax and Vitrinite Reflectance in the fore-arc basins of South-West of Ecuador}

Barba Castillo Diego Paúl.

PETROAMAZONAS EP. Quito, Ecuador.

diego_barba@petroamazonas.gob.ec

https://orcid.org/o000-0002-3091-321
Rivadeneira Montesdeoca Marco Vinicio.

Escuela Politécnica Nacional. Quito, Ecuador.

marco.rivadeneira@epn.edu.ec

https://orcid.org/0000-0001-6860-1257
Montenegro Santos Galo Vicente.

Escuela Superior Politécnica del Litoral.

Guayaquil, Ecuador.

gmontene@espol.edu.ec

https://orcid.org/oooo-00o2-5976-2179

\section{RESUMEN}

La cuantificación de Ro y Tmax son dos técnicas independientes utilizadas para caracterizar la madurez termal de las rocas. Más aún, varios estudios han demostrado que existe una relación lineal-directa entre estos dos parámetros. Para el caso de las cuencas del Sur-Oeste de Ecuador, se ha establecido una ecuación [Ro $=0.0228^{*} T \max -9.21$ ] basada en las muestras de los pozos Tiburon-0001 y B2-ANX1-1X, los cuales son considerados como los más confiables. Adicionalmente, se incorporó una zona de confianza (Tmax $\pm 1 \sigma$ ). Las muestras que caen fuera de esta zona no fueron consideradas en posteriores interpretaciones. La ecuación propuesta ha sido probada eficientemente para otros pares de datos analizados en este trabajo e incluso resulta funcional para otras cuencas. La caracterización termal del Sur-Oeste del Ecuador, da cuenta de la existencia de dos zonas que habrían alcanzado la suficiente madurez termal para generar hidrocarburos. La primera está asociada con las rocas del Cretácico tardío presentes en la Cordillera de Chongón-Colonche y a rocas del Paleoceno del Levantamiento de Santa Elena. La segunda está relacionada con rocas del Neógeno y que se encuentran afectadas por el sistema de fallas Puná-Santa Clara. La madurez termal observada en estas zonas ha sido vinculada con procesos tectónicos, ya sea por la acreción de terrenos (Sistema Cretácico tardío - Paleoceno) y al escape del Bloque Nor-Andino (Sistema Neógeno).

Palabras clave: reflectancia de la vitrinita (Ro); temperatura máxima (Tmax); temperatura de soterramiento (T-burial); madurez termal, roca madre, cuenca de ante-arco.

\section{ABSTRACT}

Quantification of Ro and Tmax are two independent techniques used to constrain the thermal maturity of rocks. Furthermore, several studies have recognized a direct linear relationship between both parameters. In the case of the fore-arc basins of the South-West of Ecuador, we have established an equation [Ro $=0.0228^{\star}$ Tmax -9.21$]$ based in the data of samples of the Tiburon-0001 and B2-ANX1$1 X$ wells; which correspond to the best quality data. Additionally, a confidence zone was established (Tmax $\pm 1 \sigma)$. The samples that fall out of this zone were not considered in further interpretations. The proposed equation has been tested efficient for other pairs of data analyzed in this work and for other basins. The thermal characterization of the South-West of Ecuador shows that two zones may have 
reached sufficient thermal maturity in order to generate hydrocarbons. The first one is related with late Cretaceous rocks of the Chongón-Colonche Range and with Paleogene rocks of Santa Elena High. The second one is related with Neogene rocks affected by the Puna-Santa Clara fault system. The thermal maturity observed in these zones has been linked with tectonic processes, due to accretionary events (Late Cretaceous and Paleogene systems) and to the Nor-Andean Block scape (Neogene system).

Keywords: vitrinite reflectance (Ro); maximum temperature (Tmax); burial temperature (T-burial); thermal maturity, source rock, fore-arc basin.

\section{INTRODUCCIÓN}

La caracterización de la madurez termal de una roca madre es el primer paso en el análisis de cuenca, ya que determina si una roca madre entró o no en ventana de generación de hidrocarburos y por lo tanto determina la existencia de un sistema petrolífero.

Una roca madre puede estar ampliamente distribuida en una cuenca sedimentaria; sin embargo, sólo en ciertos lugares habría alcanzado la madurez termal. Una roca madre pudo haber alcanzado una o varias veces la ventanas de generación. La zona donde la roca madre se encuentra en ventana de generación se denomina cocina. La cocina se denomina activa si la roca madre se encuentra actualmente en ventana de generación, para lo cual se utiliza el gradiente geotermal actual de la cuenca; por otro lado, se denomina paleo-cocina al lugar donde la roca madre entró en ventana de generación en el pasado geológico, para lo cual se utilizan geotermómetros, uno de los más utilizados es la reflectancia de vitrinita.

En la fase inicial de exploración de una cuenca, las muestras analizadas corresponden a los afloramientos. En una segunda etapa se busca tomar muestras representativas de la roca madre, ya sea con campañas de geología de superficie más detalladas o a través de sondeos de perforación (Core Hole), en este caso se busca que las rocas no presenten meteorización, que afecta a la calidad de la roca. Finalmente, en un estado avanzado de exploración, se toman muestras de los pozos exploratorios, ya sean a través de un núcleo continuo, núcleos de pared (Side-wall Core) o ripios de perforación (Ditch samples). En general, resulta difícil contar con muestras de la parte más profunda de la cuenca (depo-centro), por lo que usualmente se extrapolan los datos conocidos a la zona más profunda mediante mapas estructurales, los que han sido creados a partir de secciones de sísmica de reflexión o modelados a partir de encuestas gravimétricas/magnetométricas.

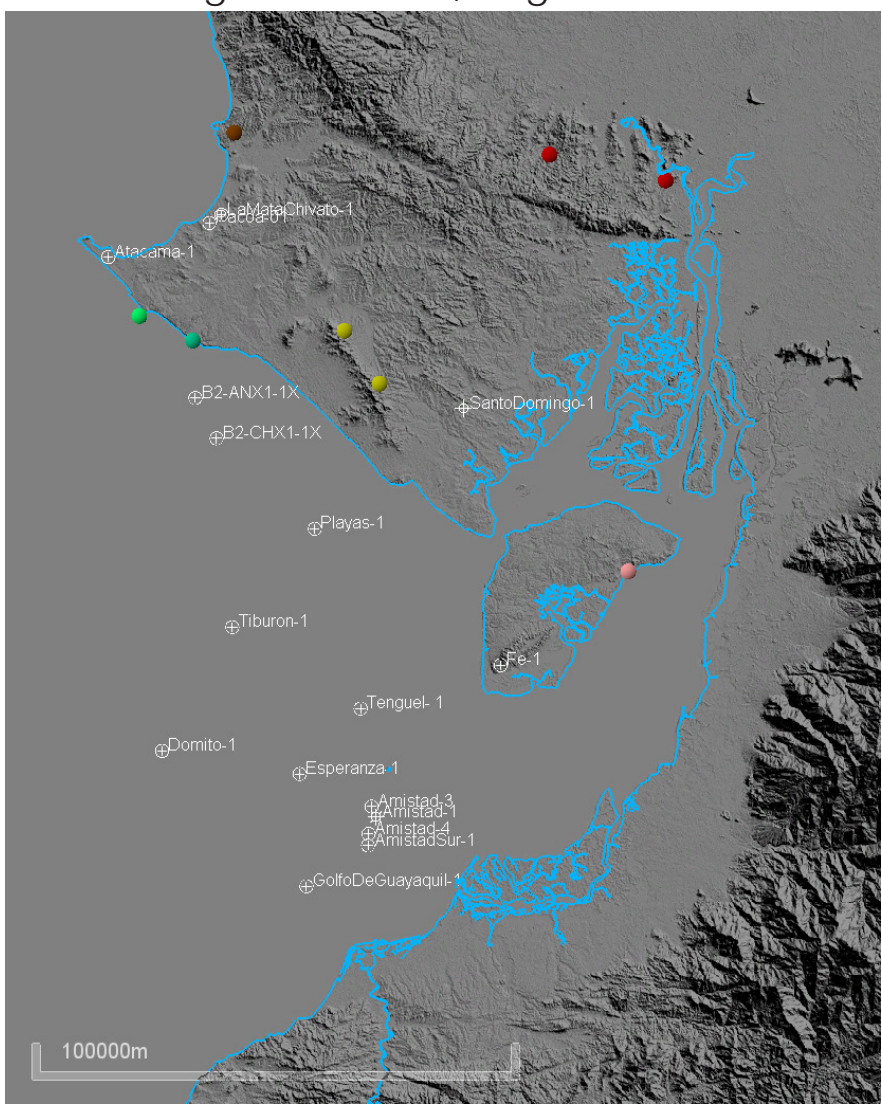

Figura.1 Ubicación de los lugares de muestreo. La mayor cantidad de muestras proviene de los perfiles geoquímicos de los pozos exploratorios.

La madurez termal rutinariamente suele ser caracterizada por dos técnicas analíticas independientes; estas son la medición de la temperatura máxima (Tmax) a través de la pirólisis rock-eval y la medición de la reflectancia de la vitrinita (Ro).

El uso conjunto de las medidas de Ro y Tmax, permiten caracterizar de forma adecuada si una roca entró en ventana de generación de hidrocarburos, la ausencia de uno de estos dos parámetros crea incertidumbre en la interpretación. La reflectancia de la vitrinita y la Tmax se miden en rocas clásticas de grano fino con presencia de materia orgánica diseminada. 
La coloración de las esporas (SCl, por sus siglas en inglés) y el índice de alteración termal (TAI, por sus siglas en inglés) son otras técnicas para evaluar la madurez termal de una roca madre, en ambos casos mide los cambios de color de los palinomorfos. Ambas técnicas deben ser utilizadas como métodos suplementarios, ya que son técnicas subjetivas, relacionadas a la experticia del laboratorista.

Por otro lado, el análisis de las trazas de fisión de apatito es otra técnica para medir la madurez, pero se aplica a rocas clásticas de grano grueso a medio, como las areniscas. La longitud de las trazas de fisión en los cristales de apatito está relacionada con la temperatura máxima a la que la roca ha sido expuesta y por lo tanto provee información relacionada a la historia termal. Esta técnica es poco utilizada, debido a su alto costo.

Las cuencas de ante-arco se localizan entre la zona de subducción y la cordillera de los Andes. Existen dos rocas madres aceptadas como posibles generadoras de los hidrocarburos presentes en esta región. La primera es la Fm. Calentura del Cretácico tardío, la cual está asociada con el basamento de las cuencas, esta formación se habría depositado en una cuenca marginal anóxica, la que posteriormente fue acrecionada a la margen Sudamericana. La segunda roca madre es la Fm. Dos Bocas cuya edad es Oligoceno tardío - Mioceno temprano. Adicionalmente, existen otras potenciales rocas madres que no se han podido caracterizar adecuadamente, debido a su escasa distribución en la superficie, estas rocas son de edad Eoceno y serían equivalentes a las lutitas de la Fm. Talara del Perú.

En general, mientras más antigua es una roca, mayor enterramiento habría experimentado. Por lo tanto, es común tener un incremento de la madurez termal conforme se alcanzan rocas más antiguas. Sin embargo, existen excepciones, unas asociadas con zonas que han experimentado cabalgamientos, colocando una mayor carga litostática sobre la potencial roca madre y otras asociadas a zonas de fallamiento cortical, lo que permite una mayor transferencia de calor.

La temperatura de enterramiento (T-burial) asociada a la generación de hidrocarburos, es la temperatura a la que fueron expuestos los sedimentos producto del soterramiento al que estos fueron sometidos. En general, la relación entre la reflectancia de la vitrinita y la temperatura de generación cumple una función logarítmica, como la propuesta por Barker \& Pawlewicz (1994).

\section{METODOLOGÍA}

El análisis realizado se enfoca en las cuencas del Sur-Oeste ante-arco ecuatoriano, donde se tiene la mayor cantidad de información. No incluye información de la cuenca Zorritos, ni Talara en el lado peruano.

El trabajo siguió tres pasos: 1) Recopilar y validar la información; 2) Seleccionar los mejores datos para establecer la ecuación de correlación y 3) Buscar que la ecuación definida represente a todas las muestras, tanto de nuestro estudio, como también a muestras provenientes de otras cuencas sedimentarias.

\section{Set de Datos}

El trabajo se basa en la recopilación de datos de Tmax y reflectacia de la vitrinita de las cuencas de Sur-Oeste del Ecuador generados durante varias campañas exploratorias entre los años de 1975 y 2008. La información se encuentra en la base de datos de geoquímica orgánica de la Gerencia de Exploración de PETROAMAZOMAS EP.

En general, para la medición de Tmax se utiliza un analizador Rock-Eval (e.g. Rock-Eval 6), mientras que para determinar la reflectancia de la vitrinita se utiliza un Fotómetro-Microscopio (e.g. FM Compacto-MPV).

La pirolisis es una técnica de laboratorio, donde se simula el proceso natural de maduración de la materia orgánica. El analizador de pirolisis RockEval, registra los gases generados desde la roca madre durante un proceso de calentamiento programado en ausencia de oxígeno. Esta técnica fue desarrollada por el Instituto Francés del Petróleo (IFP, por sus siglas en Francés) a finales de los años 70s, desde aquella época hasta la actualidad se ha convertido en un standard en la caracterización de la roca madre (Espitalié et al., 1977; McCarthy et al., 2001). Los principales parámetros geoquímicos que se mide son el S1, S2, S3, Tmax; dentro de estos cuatro parámetros, es de interés para este trabajo el S2 y la Tmax.

EI S2 es la cantidad de hidrocarburo pirolizable [mg HC / g de roca] presente en la roca y es un 
indicador del potencial actual de generación. La Tmax $\left[{ }^{\circ} \mathrm{C}\right]$ corresponde a la temperatura del horno en el momento de la generación máxima durante el análisis S2. El Tmax es un valor relativo del nivel de madurez termal, no representa la temperatura de enterramiento actual de la roca.

La reflectancia de la vitrinita (\%Ro) es la medida del porcentaje de luz incidente reflejada desde la superficie de la vitrinita. La limitación de esta técnica se da por la ausencia de kerógeno recuperable en una muestra, lo que la convierte en estéril para este tipo de análisis. La cantidad de luz reflejada por los macerales de la vitrinita determina la madurez termal de la roca (McCarthy et al., 2001). A partir de un análisis estadísti$\mathrm{co}$, se determina la reflectancia de la vitrinita, el histograma de frecuencia absoluta determina la calidad del análisis, así una muestra con distribución uni-modal tendrá mejor calidad de datos, mientras que una muestra bi-modal requiere una interpretación adicional para determinar cuál es la población "indígena" de la muestra, dejando de lado a la población contaminante, las que están asociadas a reelaborado (re-worked) o a derrumbes (caving) generados durante la perforación de los pozos (aplica a ripios de perforación). En histogramas con amplia dispersión, se tiene mayor grado de complejidad en la interpretación de los datos y por lo tanto mayor incertidumbre al reportar un valor representativo de Ro; por lo tanto, el histograma debe ser interpretados en un contexto regional para validar su valor.

Tabla 1. Disponibilidad de datos. Todas las muestras sin filtrar, con Ro y/o Tmax

\begin{tabular}{|c|r|c|}
\hline Muestras & Ro & Tmax \\
\hline Pozos & 361 & 452 \\
\hline Afloramientos & 39 & 33 \\
\hline TOTAL & 400 & 485 \\
\hline
\end{tabular}

La base de datos fue recolectada de informes inéditos realizados por CEPE, PETROPRODUCCION, PETROECUADOR EP y PETROAMAZONAS EP; así como también de reportes elaborados por diversas operadoras que trabajaron en esta región durante los años 70s y 80s (e.g. ADA, BELCO) y que ahora forman parte del archivo técnico de PETROAMAZONAS EP. La base de datos primaria agrupa 400 muestras con datos de reflectancia de vitrinita y 485 muestras de Tmax (Tabla 1).
Tabla 2. Disponibilidad de datos. Muestras con Ro y Tmax.

\begin{tabular}{|c|c|}
\hline Muestras & Ro y Tmax (pares) \\
\hline Pozos & 241 \\
\hline Afloramientos & 33 \\
\hline TOTAL & 274 \\
\hline
\end{tabular}

Para el caso de los pozos, las muestras fueron emparejadas buscando que los intervalos analizados ya sea por reflectancia de la vitrinita o por pirólisis, sean los mismos. Los datos emparejados de pozos y muestras de afloramiento (Tabla 2), fueron ploteados en un diagrama bidimensional (Fig. 2) para visualizar si existe relación lineal directa entre ellos, tal como ha sido identificado en otras regiones del mundo y cuyas ecuaciones han sido recopiladas por Jarvie (2018).

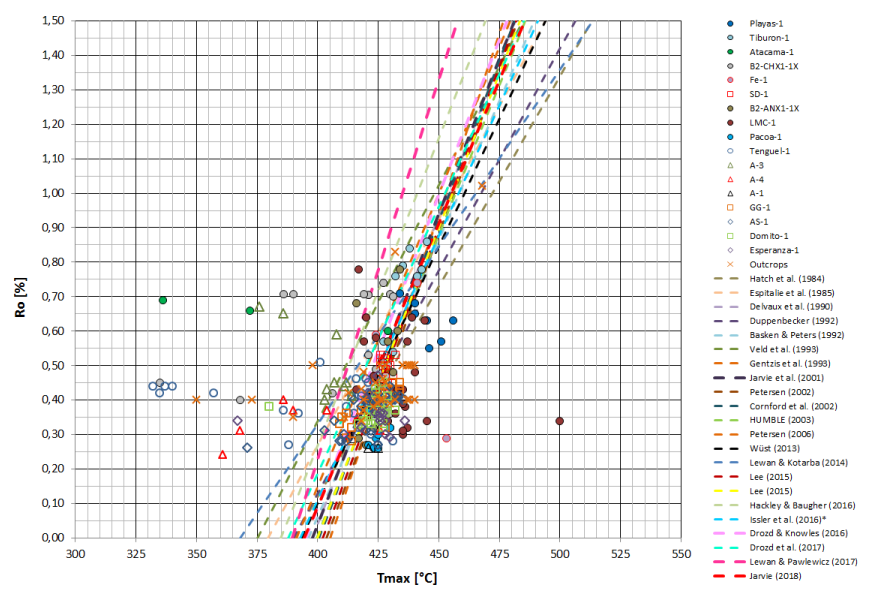

Figura. 2 Set de datos de las cuencas de ante-arco del SW del Ecuador en relación con las diferentes ecuaciones propuestas por otros autores y recopiladas por Jarvie (2018).

Issler et al. (2016) considera que la relación entre Ro y Tmax es de tipo exponencial, contradiciendo a la mayoría de las observaciones. Sin embargo, los datos de Issler et al. (2016) fueron re-ploteados y se re-calculó una ecuación lineal, la que fue incorporada en el set de curvas mostradas en la Fig. 2.

Los dos últimos estudios sobre el tema corresponden a Lewan \& Pawlewicz (2017) y a Jarvie (2018); ambos trabajos son discutidos a continuación.

Respecto a la propuesta de Lewan \& Pawlewizc (2017), vemos que no representa a nuestros datos (Fig. 2), ya que se encuentra desplazada hacia la izquierda. Adicionalmente, su coeficiente de determinación ( $R 2=0.46$ ) indica una alta dispersión de los datos y por lo tanto alta incertidumbre (Fig. 3). 


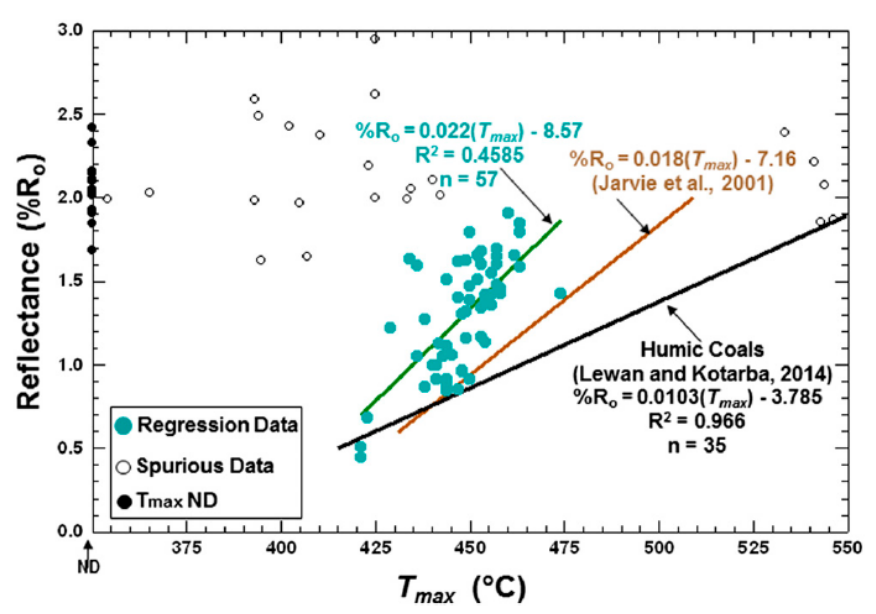

Figura. 3 Ploteo de temperatura en la máxima tasa de petróleo generado (Tmax) versus reflectancia medida de datos del USGS de las Lutitas Barnett (Lewan \& Pawlewicz, 2017).

Por otro lado, Jarvie (2018) realiza una compilación de dieciséis ecuaciones, propuestas entre 1984 hasta 2017 (Fig. 2). Asigna valores de Tmax entre 430 y $485^{\circ} \mathrm{C}$ en pasos de $5^{\circ} \mathrm{C}$; y calcula una RoE utilizando cada una de las ecuaciones; así obtuvo una matriz con 192 pares de datos, los cuales fueron ploteados y calculados la correspondiente regresión lineal. Esta técnica no es la apropiada ya que depende del rango establecido (Fig. 4). Es decir, que si ampliamos el rango observado entre 400 y $550^{\circ} \mathrm{C}$ de Tmax y entre 0.3 y $2.0 \%$ Ro, se obtiene una nueva ecuación. A pesar de que la ecuación cubre a nuestro set de datos, no se la debe utilizar, pues carece de validez matemática.

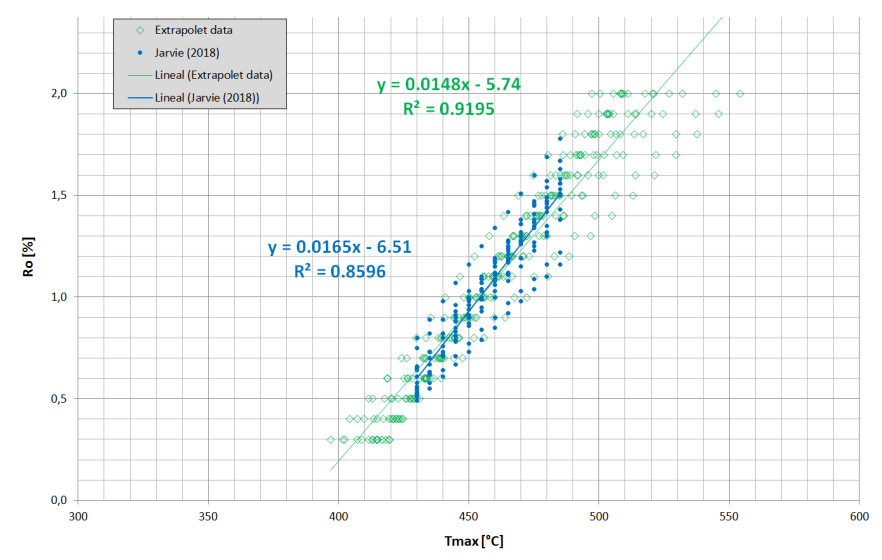

Figura. 4 Muestras con pares de datos de acuerdo a Jarvie (2018) en color azul. Muestras ampliadas su rango en color verde. Se evidencia que el rango de observación determina la ecuación de correlación, por lo que esta técnica sintética no resulta adecuada.

En el caso que un set de datos analizado no cuente con las condiciones adecuadas para calcular su propia ecuación de correlación, se debe seleccionar a la mejor ecuación de la bibliografía. En nuestro caso, funcionaría la ecuación propuesta por Gentzis et al. (1993).
Relación entre Tmax - Ro: Aproximación usando datos locales

Para poder encontrar una ecuación que relacione el Ro y la Tmax de nuestras muestras, se debió en primera instancia depurar la base de datos, pasando de 274 a 239 muestras. Las muestras eliminadas fueron aquellas que caen fuera de la tendencia regional (Spurious Data). Este es un método de discretización rápida y debe ser utilizado cuando existe gran cantidad de muestras (Fig. 2); aunque siempre es recomendable trabajar en conjunto con la petrografía del kerógeno y con los otros parámetros calculados en la pirolisis Rock-Eval (e.g. S1, S3, HI, OI), para tratar de entender de mejor manera cuál sería la causa de su desviación en relación a la tendencia de la misma cuenca y de la tendencia mundial.

Tabla 3. Disponibilidad de datos. Muestras con Ro y Tmax dentro de la tendencia mundial.

\begin{tabular}{|c|c|}
\hline Muestras & Ro y Tmax (pares validados) \\
\hline Pozos & 210 \\
\hline Afloramientos & 29 \\
\hline TOTAL & 239 \\
\hline
\end{tabular}

La alta concentración de muestras con baja madurez termal, la falta de control de las muestras de moderada madurez y la ausencia de muestras con alta madurez termal, ocasionó a que la ecuación lineal obtenida por regresión lineal no sea correcta (Fig. 5).

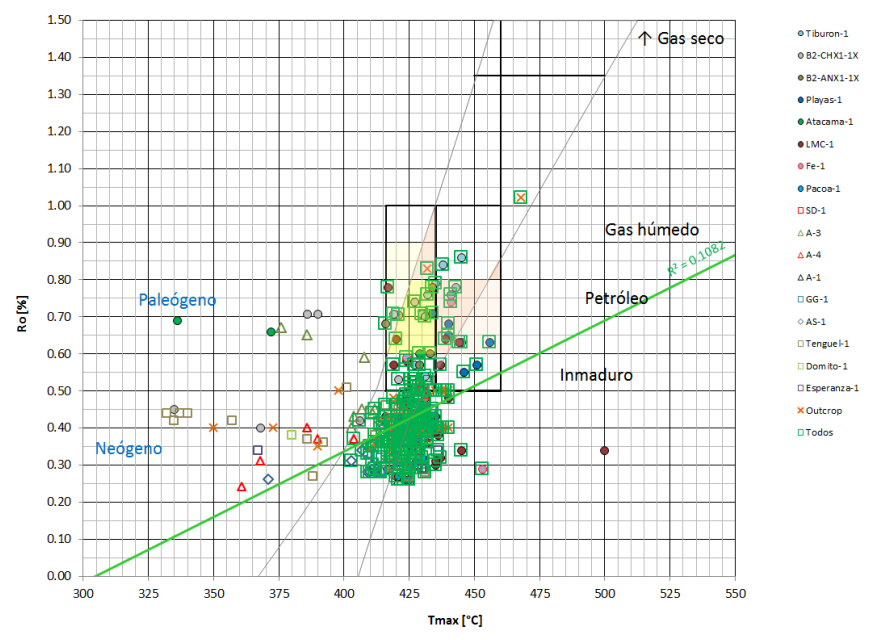

Figura. 5 Set de datos filtrados (serie en color verde), se han eliminado los valores anómalos, especialmente de Tmax. La alta dispersión de los datos no permite obtener una regresión lineal adecuada.

En este trabajo no se considera caracterizar a la roca madre desde el punto de vista de la riqueza de la materia orgánica o tipo de kerógeno. Aunque las muestras seleccionadas para realizar la pirolisis Rock-Eval son aquellas que poseen más de $0.5 \%$ de carbono orgánico total 
(TOC, por sus siglas en Inglés), es decir disponen de la cantidad de materia orgánica favorable para efectuar la pirolisis.

A la falta de obtener una buena correlación entre Tmax y Ro por regresión directa aplicada a todas las muestras, se procedió a buscar una solución alternativa. Para los cual, se seleccionaron los datos más fiables, analizando cada una de las muestras de afloramiento, así como también cada uno de los pozos. De todo el universo de muestras, se seleccionaron los pozos Tiburon-0001 y B2-ANX1-1X (Fig. 6), a los cuales se aplicó una regresión lineal y se obtuvo una ecuación, la que resulto tener una buena correlación. La ecuación propuesta se encuentra dentro de las observaciones propuestas en trabajos anteriores (Figs. 2 y 5).

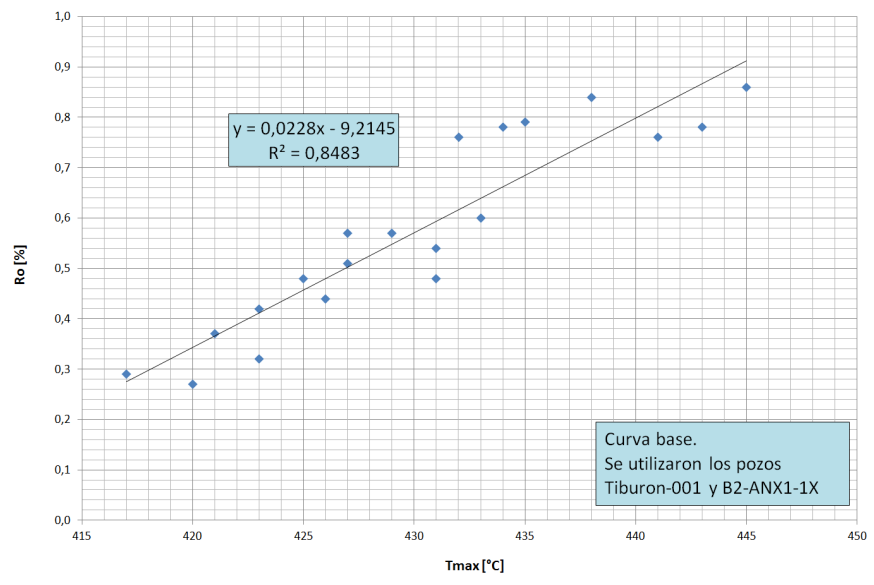

Figura. 6 Correlación realizada entre Ro y Tmax para dos pozos con excelentes medidas. Localización de los pozos en la Figura 1.

La ecuación obtenida (Ec. 1) servirá para calcular la reflectancia de la vitrinita equivalente (RoE), en el caso de disponer únicamente datos de Tmax.

Ro $=(0.0228 * \operatorname{Tmax})-9.21$

Validación gráfica de la ecuación propuesta

La ecuación propuesta (Ec.1) fue comparada (QC visual) con el resto de muestras del Sur-Oeste ecuatoriano; así también con muestras de otras cuencas de ante-país de Ecuador, Colombia (Hernández, 1998) y Perú (Calderón et al., 2018). También se la comparó con la cuenca de Sandino (Struss et al., 2008) en Nicaragua, por presentar similares condiciones geológicas. Finalmente, se montaron los datos de la cuenca de Maracaibo (Mongenot et al., 1996) por disponer de datos de alta madurez. En todos los casos, la Ecuación (1), los repre-

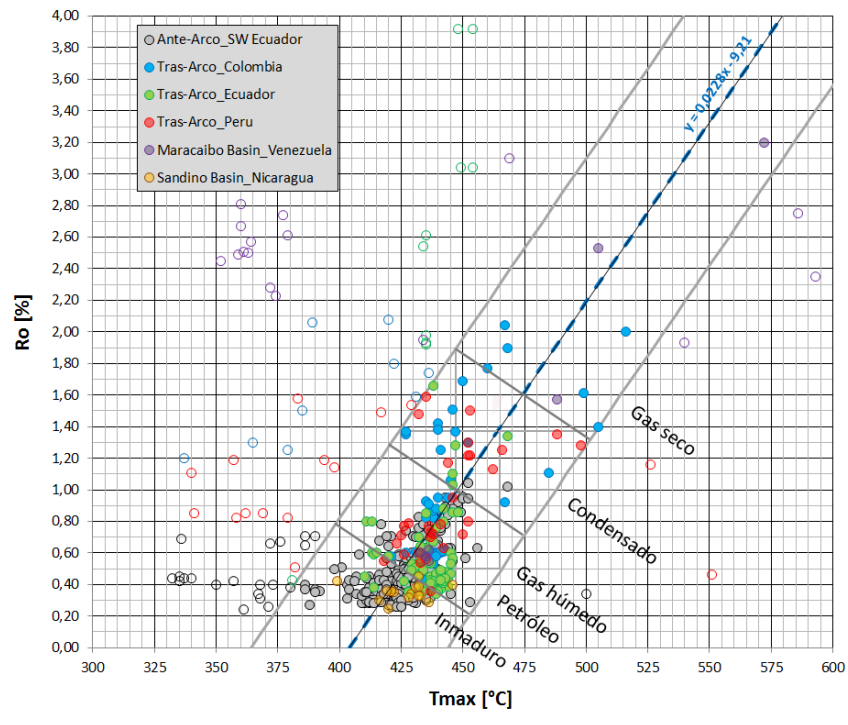

Figura. 7 Validación de la ecuación propuesta. Se incorporaron datos de las cuencas de ante-país de Colombia, Ecuador y Perú; así como de las cuencas de Sandino en Nicaragua y Maracaibo en Venezuela. Adicionalmente se incorporó una banda de tolerancia $(\operatorname{Tmax} \pm 1 \sigma)$, los datos que caen fuera de dicha banda no deberían ser utilizados en la caracterización termal de la roca madre (marcadores sin relleno).

Por último y una vez determinada una ecuación de correlación representativa, se procedió a establecer una banda de confidencia, la cual fue construida al adicionar y restar una desviación estándar ( $\pm 1 \sigma$ ) del Tmax (Fig. 7). Las muestras que caen por fuera de esta banda, son consideradas como erróneas y por lo tanto no aptas para la caracterización termal de la muestra y por consiguiente no deben ser tomadas en cuenta para la interpretación a nivel de unidad geológica y cuenca.

Existe una considerable cantidad de muestras que presentan altos valores de reflectancia de vitrinita, pero bajos valores Tmax; estas muestras son consideradas como erróneas (Fig. 7). Se requiere un análisis petrográfico detallado de la materia orgánica presente en las muestras para determinar la causa de su desviación.

\section{DISCUSIÓN}

En la figura 8 se muestra las diferencias entre la Tmax medida por pirolisis (Rock-Eval_Temp) y la Tmax calculada a partir de la reflectancia de la vitrinita (Model_Temp) usando la Ecuación (1), el valor ploteado corresponde al valor promedio de todas las muestras que disponen de Tmax y Ro, para cada edad geológica (ver Tabla 3). Existe una marcada diferencia entre las paleo-temperaturas del Cretácico tardío Paleoceno (e.g. Fm. Calentura; Fm. Azúcar), 
respecto al Eoceno-Reciente. Estas diferencias también fueron delineadas en el mapa del gradiente geotermal realizado por Barba (2017), aunque en sentido contrario, ya que la Fm. Azúcar en el levantamiento de Santa Elena muestra un gradiente geotermal extremadamente bajo. La Fm. Azúcar no ha sido reconocida en otras partes del litoral ecuatoriano. La alta madurez termal de la Fm. Azúcar puede estar relacionada con el proceso de acreción ocurrido hace 50 Ma.

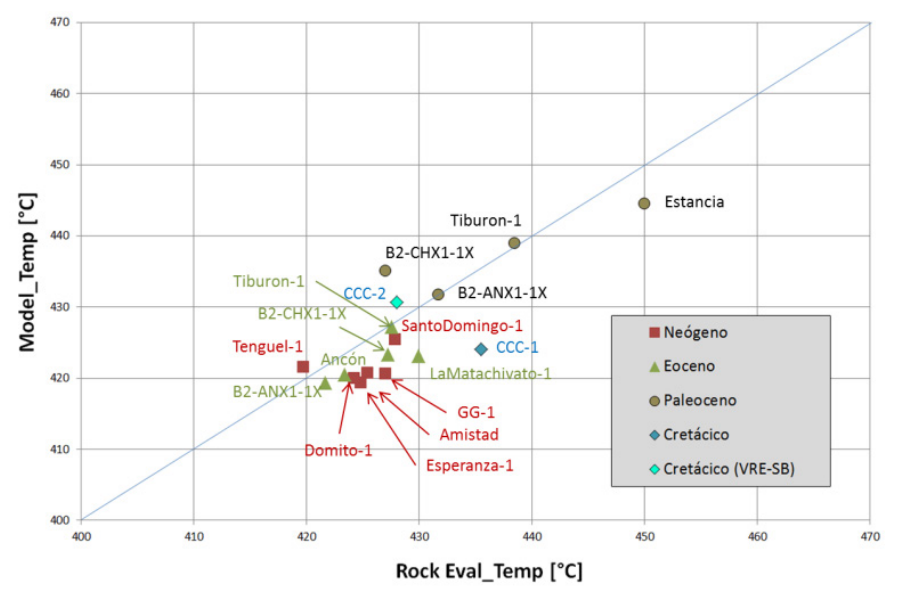

Figura. 8 Correlación entre los datos modelados y las medidas reales de Tmax. Se han ploteado los valores promedios de las unidades geológicas presentes en el área. Fm. Calentura: CCC-1 = utilizando Ro; CCC-2 = utilizando VRE-SB.

Las unidades del Eoceno (e.g. pozos Tiburon-1, B2-CHX1-1X, La Matachivato-1, B2-ANX1-1X, afloramientos de Ancón) y del Neógeno (e.g. pozos Golfo de Guyaquil-1, Amistad-1, Amistad-4, Esperanza-1, Domito-1, Tenguel-1) se encuentran en una etapa inmadura de generación de hidrocarburos (Fig. 8), este comportamiento es similar al observado en la cuenca Sandino, costa afuera al Oeste de Nicaragua (Struss et al., 2008).

La Fm. Calentura tiene un promedio de Tmax de $436{ }^{\circ} \mathrm{C}$ ( $\operatorname{mín}=427^{\circ} \mathrm{C}$; máx $=440{ }^{\circ} \mathrm{C}$ ), lo que indica que habría entrado en ventana de generación temprana de petróleo. Por otro lado, la reflectancia de la vitrinita reportada tiene un promedio de $0.46 \%$ (mín $=0.40 \%$; máx $=0.50$ $\%$ ), según este parámetro, esta roca madre no habría entrado en ventana de generación. La materia orgánica que se encuentra presente en las rocas de la Fm. Calentura es de tipo algácea (Kerógeno sapropélico), con ausencia de vitrinita; por lo tanto los valores reportados como Ro no son correctos. En una muestra de la Fm. Calentura se obtuvo una reflectancia del bitumen sólido (RSB, por sus siglas en Inglés) de $0.35 \%$, que equivale a una reflectancia de la Vitrinita (VRE-SB) de $0.61 \%$, de acuerdo a French et al. (2020). Este valor de reflectancia de la Vitrinita es concordante con la Tmax obtenida de los ensayos de pirólisis Rock-Eval (Fig. 8).

Las temperaturas de enterramiento máximo (T-burial) fueron calculadas utilizando la Ecuación (2); siguiendo la aproximación de Barker \& Pawlewicz (1994).

T-burial $=[\ln (\mathrm{Ro})+1.68] / 0.0124$

En un perfil geoquímico se colocan las T-burial calculadas (Fig. 9) a partir de la reflectancia de la Vitrinita, así se puede determinar el paleo-gradiente de una cuenca sedimentaria. En el caso de no disponer de datos de Ro, se debe utilizar la VRE derivada a partir de la Tmax (VRE-Tmax).

El gradiente geotermal actual y el paleo-gradiente son dos de los insumos necesarios para delinear la ubicación de las cocinas de hidrocarburos. Los otros dos insumos corresponden a la caracterización de la roca madre (tipo de Kerógeno, Potencial generador, etc.) y los mapas estructurales (Tope y Base o al menos un mapa de un marcador cercano). No toda roca madre llega a ser efectiva, ya que puede tener la suficiente materia orgánica, pero no haber alcanzado la temperatura de generación; o pudo haber entrado en ventana de generación, pero ser una roca pobre en calidad o cantidad de materia orgánica. Con toda esta información, se puede dar el siguiente paso en la modelización de los procesos de generación y expulsión del sistema petrolífero.

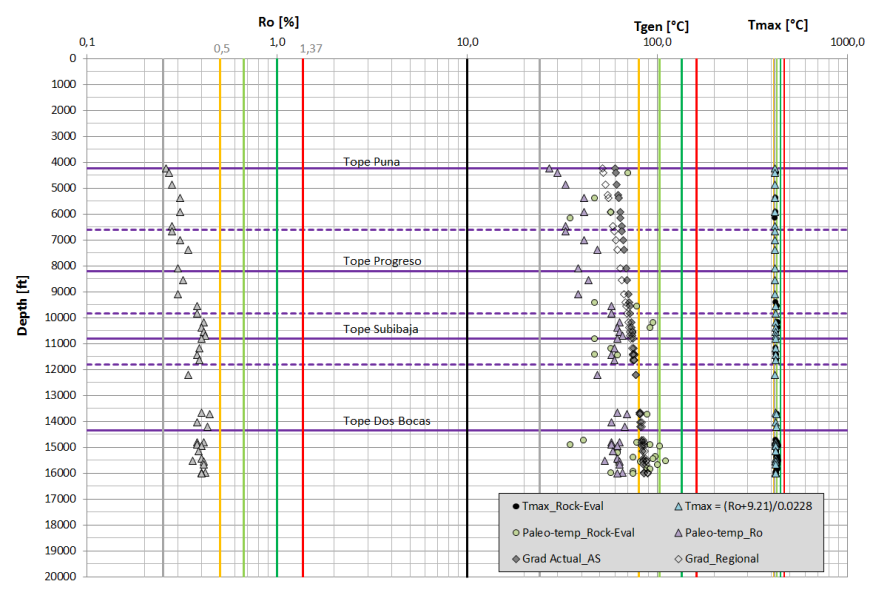

Figura. 9 Perfil geoquímico del pozo Amistad Sur-0001. Se evidencia que la Fm. Para comparación se ha añadido el gradiente geotermal actual del pozo y el gradiente actual promedio de la región, de acuerdo a las estimaciones realizadas por Barba (2017). 
Antes de proceder con la caracterización termal de una roca madre, se debe realizar una validación de los datos utilizando patrones mundiales, los datos que no sigan una tendencia conocida, son considerados como anómalos; si no existe una explicación de su desviación, deben ser considerados como erróneos.

Para calcular la ecuación de correlación se deben utilizar únicamente los mejores datos, caso contrario se puede inducir un error. En el presente trabajo se utilizaron los datos de Tmax y Ro de los pozos Tiburon-0001 y B2ANX1-1X, los cuales son considerados como los más idóneos para el área de estudio.

La curva propuesta logra representar a otros pares de datos, tanto de la misma región como de otras regiones (e.g. cuencas de Colombia, Ecuador, Perú, Nicaragua y Venezuela).

La ecuación de correlación permite complementar el set de datos inicial. El modelo predictivo propuesto ayuda en la caracterización termal de las muestras que sólo cuenten con Tmax ó Ro.

Para la interpretación se deben utilizar los datos que caen en la zona validada por la ecuación propuesta, cuyos límites corresponden a Tmax $\pm 1 \sigma$. Esta metodología constituye una forma rápida de realizar una discretización de grandes cantidades de datos (data screening).

Para una correcta interpretación de los datos, se debe considerar la petrografía del kerógeno; como es el caso de la Fm. Calentura. En este ejemplo, la ausencia de vitrinita provoca que los valores de Ro sean incorrectos; debiéndose recurrir a otras técnicas, como por ejemplo la reflectancia del bitumen sólido, para posteriormente calcular su correspondiente reflectancia de la vitrinita equivalente (VRE-SB).

La caracterización termal del Sur-Oeste del Ecuador, da cuenta de la existencia de dos zonas que habrían alcanzado la suficiente madurez termal para generar hidrocarburos. La primera está asociada con las rocas del Cretácico tardío presentes en la Cordillera de
Chongón-Colonche y a rocas del Paleoceno del Levantamiento de Santa Elena. La segunda está relacionada con rocas del Neógeno y que se encuentran afectadas por el sistema de fallas Puná-Santa Clara. La madurez termal observada en estas zonas ha sido vinculada con procesos tectónicos, ya sea por la acreción de terrenos (Sistema Cretácico tardío - Paleoceno) y al escape del bloque Nor-Andino (Sistema Neógeno). La escasa distribución areal de las cocinas conlleva a un limitado potencial generador de las rocas madres involucradas.

\section{AGRADECIMIENTOS}

Agradecemos a la gerencia nacional de operaciones de PETROAMAZONAS EP quien ha permitido la publicación de los resultados generados durante la actualización de los diferentes modelos geológicos efectuada por la gerencia de exploración. Con este trabajo otorgamos un reconocimiento a los ex-colegas del Centro de Investigaciones Geológicas de Guayaquil (CIGG, ex-LABOGEO), quienes durante varias décadas ayudaron con los análisis geoquímicos y petrográficos de las diferentes campañas exploratorias efectuadas por CEPE, PETROPRODUCCION, PETROECUADOR EP y PETROAMAZONAS EP. Este trabajo formó parte del curso de Metodología para la Elaboración y Presentación de Artículos Técnicos dictado por FIGEMPA-IGAPO-PAM EP.

\section{REFERENCIAS}

Barba, D. (2017). Gradiente Geotermal de las Cuencas de Ante-arco del Ecuador. Resúmenes extendidos de las VIII Jornadas en Ciencias de la Tierra, EPN, 5 p.

Barker, C., Pawlewicz M. (1994). Calculation of Vitrinite Reflectance from Thermal Histories and Peak Temperatures - A Comparison of Methods, in: Mukhopadhyay, P., Dow, W. (Eds.), Vitrinite Reflectance as a Maturity Parameter. American Chemical Society, 216229.

Calderón, Y., Baby, P., Vela, Y., Hurtado, C. Eude, A., Roddaz, M., Brusset, S., Calvès, G., and Bolaños, R. (2017). Petroleum Sys- 
tems Restoration of the Huallaga-Marañon Andean Retroforeland Basin, Peru, in Mahdi A. AbuAli, Isabelle Moretti, and Hege M. Nordgård Bolås, eds., Petroleum Systems Analysis-Case Studies: AAPG Memoir 114, p. 95-116.

Espitalié, J., Laporte, L., Madec, M., Marquis, F., Leplate, P., Pault, J., Boutefeu, A. (1977). Methode rapid de caracterisation des rock meres, deleur potential petrolier et leurdegre devolution. Rev. Inst. Fr. Petrol., 32, 23-42.

Grobe, A., Urai J., Littke, R., Lünsdorf, N. (2016). Hydrocarbon generation and migration under a large overthrust: The carbonate platform under the Semail Ophiolite, Jebel Akhdar, Oman. International Journal of Coal Geology, Volume 168, Part 1, 3-19.

French, K., Birdwell, J., Lewan, M. (2020). Trends in thermal maturity indicators for the organic sulfur-rich Eagle Ford Shale. Marine and Petroleum Geology, http://doi.org/10.1016/j.marpetgeo.2020.104459.

Gentzis, T., Goodarzi, F., Snowdon, L. (1993). Variation of maturity indicators (optical and Rock-Eval) with respect to organic matter type and matrix lithology: an example from Melville Island, Canadian Arctic Archipelago, Marine and Petroleum Geology, v. 10, p. 507-513.

Hernández, V. (1998). Evaluación Potencial Generador Cordillera Oriental. http://recordcenter.sgc.gov.co/B3/12005000520702/ documento/pdf/0101207021101000.pdf.

Issler, D. Jiang, C., Reyes, J., Obermajer, M. (2016). Integrated analysis of vitrinite reflectance, Rock-Eval 6, gas chromatography, and gas chromatography-mass spectrometry data for the Reindeer D-27 and Tununuk K-10 wells, Beaufort-Mackenzie Basin, northern Canada. Geological Survey of Canada, Open File 8047, 94 p.

Jarvie, D., Claxton, B., Henk, F., Breyer, J. (2001). Oil and shale gas from the Barnett Shale, Fort Worth Basin, Texas (abs.): AAPG Annual Convention, Denver, Colora- do, June 3-6, 2001, accessed March 28, 2017.

Jarvie, D. (2018). Correlation of Tmax and Measured Vitrinite Reflectance, TCU Energy Institute Notes, $13 \mathrm{p}$.

Lewan, M. \& Pawlewicz, M. (2017). Reevaluation of thermal maturity and stages of petroleum formation of the Mississippian Barnett Shale, Fort Worth Basin, Texas. AAPG Bulletin, v. 101, No. 12, 1945-1970.

McCarthy, K., Rojas, K., Niemann, M., Palmowski, D., Peters, K., Stankiewicz, A. (2011). Basic Petroleum Geochemistry for Source Rock Evaluation. Oilfield Review Summer, Schlumberger 23, No. 2, 12 p.

Mongenot, T., Tribovillard, N.-P., Desprairies, A., Lallier, E., Laggoun, F. (1996). Trace elements as palaeoenvironmental markers in strongly mature hydrocarbon source rocks: the Cretaceous La Luna Formation of Venezuela. Sedimentary Geology, 103, 2337.

Struss, I., Artiles, V., Cramer, B., Winsemann, J. (2008). The Petroleum System in the Sandino Forearc Basin, Offshore Western Nicaragua. Journal of Petroleum Geology, v. 31(3), 221-244. 